\title{
Morfología del Velo Palatino en Individuos Fisurados Opera- dos, y su Relación con el Grado de Insuficiencia Velo Faríngea
}

\author{
Palate Velum Morphology on Operated Cleft Palate Patients and \\ Its Relation with the Degree of Pharyngeal Velum Insufficiency
}

Arnoldo Hernández Caldera",**; Miguel Muñoz Domon ${ }^{* *}$; Carolina Salazar Ponce** \& Noemi Leiva Villagra**

HERNANDEZ, C. A; MUÑOZ, D. M; SAlaZAR, P. C. \& LEIVA, V. N. Morfología del velo palatino en individuos fisurados operados, y su relación con el grado de insuficiencia velo faríngea. Int. J. Morphol., 31(1):87-93, 2013.

RESUMEN: La fisura labio palatina corresponde a una malformación congénita, caracterizada por la interrupción en la continuidad de los tejidos. La cirugía primaria del velo del paladar permite reconstituir la morfología perdida del velo palatino, sin embargo ésta no siempre considera la devolución de la anatomía del músculo de la úvula, por lo cual, el velo operado adquiere una anatomía anómala impidiendo el cierre óptimo entre cavidad oral y nasal; esta condición se conoce con el nombre de insuficiencia velo faríngea. El objetivo de este trabajo fue asociar la morfología del velo palatino con la insuficiencia velo faríngea en individuos fisurados operados. Se realizó un estudio analítico, teniendo como unidad de muestra al velo del paladar; La muestra se seleccionó entre aquellos pacientes que cumplían con los criterios de inclusión. A los individuos seleccionados se les realizó un examen clínico y un registro fotográfico con el objetivo de clasificar la anatomía del velo del paladar. Posteriormente se les realizó una evaluación fonoaudiológica para determinar el grado de insuficiencia velo faríngea. Los datos obtenidos fueron tabulados y evaluados estadísticamente. La clasificación morfológica del velo del paladar determino que el 52\% de los velos fueran clasificados como cóncavos, los velos convexos y planos se distribuyeron ambos en un 24\%. Según la evaluación velo faríngea, el 12\% se presentaron como insuficientes, el 52\% como límite insuficiente, el 24\% como límite suficiente y tan solo el $8 \%$ como suficientes. Al relacionar la morfología del velo del paladar con evaluación velo faríngea se obtuvo un $\mathrm{p}=0,3$. Existe una variabilidad tanto en la morfología del velo del paladar como en la evaluación de la insuficiencia velo faríngea en individuos fisurados operados, siendo esta ultima desfavorable. No se estableció una relación estadística entre morfología del velo del paladar y la evaluación velo faríngea.

PALABRAS CLAVE: Fisura labio palatina; Anatomía velo-palatina; Evaluación velo- faríngea.

\section{INTRODUCCIÓN}

La fisura labio palatina corresponde a una malformación congénita, caracterizada por la interrupción en la continuidad de los tejidos del labio superior, reborde alveolar, paladar óseo y mucosa palatina de forma lateral o bilateral, sumado a la interrupción del paladar blando en su línea media (Nyberg et al., 2010; Nanda et al., 2009; Rogers et al., 2008).

Esta malformación tiene su origen en la no fusión del proceso nasal medio con los procesos maxilares durante la etapa embrionaria de la formación facial, lo cual traerá como consecuencia, una serie de modificaciones óseas, del tegumento y de las inserciones musculares ya sea a nivel labial como a nivel del velo del paladar. La disminución de la funcionalidad de las fibras musculares afectadas, determinaran una serie de alteraciones ya sea en el desarrollo del complejo maxilar, asimetría en el crecimiento de la base craneal, alteraciones en la respiración, deglución, fonoarticulación, hipoacusia y aumento en la prevalencia de infecciones del territorio máxilo facial (da Silva et al., 2010; Kwan et al., 2011).

La etiología de esta condición ha sido explicada como multifactorial, en donde los factores genéticos (Stuppia et al., 2011; Carinci et al., 2007) adquieren gran relevancia en la heredabilidad de la fisura en individuos pertenecientes a familias con mas de un caso dentro de su genealogía y con un fuerte componente étnico asociado a etnias mongoloides (Palomino et al., 2007; Ministerio de Salud, 2009). La exposición a teratógenos y a otras enfermedades y condiciones durante el embarazo, sumado a la edad materna serían

\footnotetext{
* Programa de Anatomía y Biología del Desarrollo, Facultad de Medicina y Facultad de Odontología, Universidad de Chile, Santiago, Chile.

**Instituto de Rehabilitación de Malformaciones Deformaciones MáxiloFaciales (IRMADEMA), Facultad de Odontología, Universidad de Chile, Santiago, Chile.
} 
otros factores a considerar dentro de la patogénesis de la enfermedad (Rojas et al., 2000; Shaw et al., 2003; Lorente et al., 2003; Pardo et al., 2003).

Se estima que en Chile, existe una frecuencia para esta condición de aproximadamente $1,8 \times 1000$ nacimientos de niños vivos, siendo ésta casi el doble de la tasa mundial; lo que representa evidentemente un problema de salud pública, cuyo tratamiento integral ha sido incluido dentro de las patologías GES (Ministerio de Salud). La alta prevalencia de esta malformación en Chile se debe fundamentalmente a la condición étnica de la población y a otros factores tales como la exposición a pesticidas, metales pesados y a la condición de altura de algunas ciudades en el norte del país (Sepúlveda Troncoso et al., 2008).

El velo del paladar corresponde a una entidad anatómica conformada por un esqueleto aponeurótico al cual se le insertan una serie de estructuras musculares de forma arciforme y otros de tipo tubular y de orientación anteroposterior como lo es el músculo de la úvula (Rouvière \& Delmas, 2005). El velo del paladar se ubica en la región posterior y superior de la cavidad oral y desde ese punto se proyecta hacia las regiones de las fauces y de la orofaringe. Su función principal es la de obliterar de forma hermética la comunicación que existe entre la rino y la orofaringe durante la deglución. Para realizar esta acción será necesaria la activación de forma coordinada de la musculatura velar y faríngea para de este modo horizontalizar el velo palatino con el consiguiente contacto con la pared posterior de la faringe, específicamente en una zona de solevantamiento de la misma, que anatómicamente corresponde al arco anterior del atlas (Latarjet \& Ruiz-Liard, 1995; Nanda et al.; Rouvière \& Delmas).

La morfología normal del velo del paladar al examen intraoral y producto fundamentalmente de la disposición del músculo de la úvula será observado cuando éste se encuentra de forma pasiva como una estructura de forma convexa cuyo grado de convexidad varía entre los distintos individuos y que se dispone de forma oblicua desde superior a inferior y desde anterior a posterior (Latarjet \& RuizLiard; Rouvière \& Delmas). En cuando a la simetría del mismo este también puede variar, pudiendo estar desplazado hacia uno u otro lado.

Dentro de los procedimientos terapéuticos para la resolución y mejoramiento de esta condición se encuentra la cirugía primaria de paladar y de velo del paladar cuyo objetivo es reconstituir la morfología normal del velo volviendo a reunir la musculatura y mucosa que se vio interrumpida producto de la no función de los procesos palatinos (Cortés Araya et al., 2004; Ministerio de Salud; Mulliken et al., 2003; Nyberg et al.).
Producto de la cirugía primaria realizada en el velo, éste adquiere una morfología aberrante en donde se advierte una tensión excesiva del músculo palatogloso y palatofaríngeo y una modificación en la forma convexa característica del velo palatino. Esta neomorfología del velo producto de una cirugía deficiente se caracteriza por un recogimiento y desplazamiento del músculo de la úvula y en algunos casos su bifurcación. Lo anteriormente expuesto determinara un trastorno de la función velar, que han sido agrupados con el nombre de insuficiencias velo faríngeas (Ministerio de Salud; Ysunza et al., 2002).

Un porcentaje importante de los individuos fisurados que reciben cirugía primaria del velo del paladar, desarrollan una insuficiencia velo faríngea y por ende una serie de alteraciones fonoaudiológicas, sin embargo, otros individuos no la desarrollaran pese a ser sometidos al mismo procedimiento quirúrgico. Los resultados morfológicos de la técnica quirúrgica primaria de velo palatino estarían implicados en el desarrollo de insuficiencias velo faríngeas y por ende en alteraciones de la fonoarticulación. Establecer una clasificación anatómica del velo palatino y conocer cual es el grado de insuficiencia velo faríngea que éste desarrolla, mejorará los protocolos quirúrgicos y complementará el examen diagnóstico fonoaudiológico.

\section{MATERIAL Y MÉTODO}

Se realizó un estudio analítico no experimental de casos y controles en las clínicas del Instituto de rehabilitación de malformaciones y deformaciones maxilofaciales (IRMADEMA) de la Facultad de Odontología de la Universidad de Chile.

La unidad de muestra evaluada quedó determinada por velos palatinos; el tamaño muestral se estableció por conveniencia, siendo éste de 25 . La muestra fue seleccionada entre aquellos pacientes que asistieron a tratamiento multidisciplinario a la clínica del Instituto entre los meses de marzo a diciembre del año 2011 y que cumplían con los siguientes criterios de inclusión: Hombres y mujeres entre 6 y 30 años de edad, individuos afectados por fisura velo palatina operada de forma primaria y, ausencia de aparatos de ortodoncia que cubran el paladar. Los criterios de exclusión para formar parte del grupo muestral fueron: Individuos con fisura palatina asociada a algún síndrome, individuos afectados por fisura velo palatina operada de forma secundaria e, individuos afectados por fisura velo palatina intervenidos con faringoplastía.

Para la realización de este trabajo se contó con la 
debida autorización por escrito de los pacientes que participaron voluntariamente en este estudio. En caso de menores de edad la autorización fue otorgada por el apoderado a cargo del niño.

Los instrumentos de medición utilizados para la selección fueron: La autorización del paciente, el examen intraoral y su ficha odontológica.

A los individuos seleccionados se les realizó el examen intraoral y en paralelo se procedió a tomar una fotografía clínica intraoral del velo palatino cuando éste se encuentra de forma pasiva. Para ello se utilizó cámara fotográfica digital semiprofesional Fujifilm S4000. Esta fotografía fue tomada por un único operador y se estandarizado de modo tal que fueran tomadas a la altura de la comisura y de forma paralela a la bisectriz entre el plano oclusal superior e inferior en apertura oral pasiva.

Una vez realizada las maniobras antes descritas, se procedió a realizar al análisis clínico y fotográfico con el objetivo de establecer la morfología del velo palatino para su posterior clasificación. Los datos obtenidos se registraron en una ficha ad-hoc previamente confeccionada.

De este modo se clasificó a los velos palatinos de los individuos operados en 3 grupos según lo observado en la clínica y apoyados en los registros fotográficos, estableciéndose los siguientes grupos: a) Velo palatino convexo (Fig. 1); b) Velo palatino plano (Fig. 2) ; c) velo platino cóncavo (Fig. 3).

Posterior a esto los individuos seleccionados se les evaluó el grado de insuficiencia velo faríngea por una única examinadora fonoaudióloga. El examen fonoaudiológico siguió el protocolo de evaluación de insuficiencia velo faríngea establecido por las guías clínicas de la fisura palatina del Ministerio de Salud GES 2007. Los aspectos a evaluar dentro del protocolo de evaluación de insuficiencia velo faríngea son los items emisión nasal, presión intraoral, movimientos faciales, resonancia de la voz y competencias articulares, las cuales fueron categorizadas, adjudicándoseles un determinado puntaje según protocolo. La suma total obtenida de cada uno de los items determino el grado de insuficiencia velo faríngea. Si el puntaje obtenido es de 0 el mecanismo velo faríngeo fue clasificado como suficiente; si el puntaje se encuentra entre 0 y 2 el mecanismo velo faríngeo se clasifico como limite suficiente; entre 3 y 6 puntos se clasificó como mecanismo límite insuficiente y si el puntaje obtenido es mayor a 7, los individuos fueron clasificados con un mecanismo velo faríngeo insuficiente.

Los datos obtenidos en relación a la clasificación morfológica de velo palatino y al grado de insuficiencia velo faríngea, fueron tabulados y posteriormente sometido al test estadístico Kruskal Wallis en el software STATA 11 con el objetivo de establecer la relación entre morfología del velo palatino y grado de insuficiencia velo faríngea.

\section{RESULTADOS}

Se evaluaron un total de 25 individuos, los que posterior a esto fueron incorporados a los procedimientos terapéuticos ortodónticos y fonoaudiológicos del instituto de rehabilitación de malformaciones y deformaciones máxilo faciales (IRMADEMA) de la Facultad de Odontología de la Universidad de Chile. La edad promedio de los individuos participantes en el estudio fue 16 años, teniendo estos entre 8 y 30 años de edad. La distribución por sexo quedo establecida del siguiente modo; 12 correspondieron a individuos de sexo femenino y 13 a individuos de sexo masculino. En relación a las características morfológicas de los individuos en estudio se pudo establecer que el 76\% (19 casos) presentó fisura labio-palatina unilateral operada; el 16\% (4

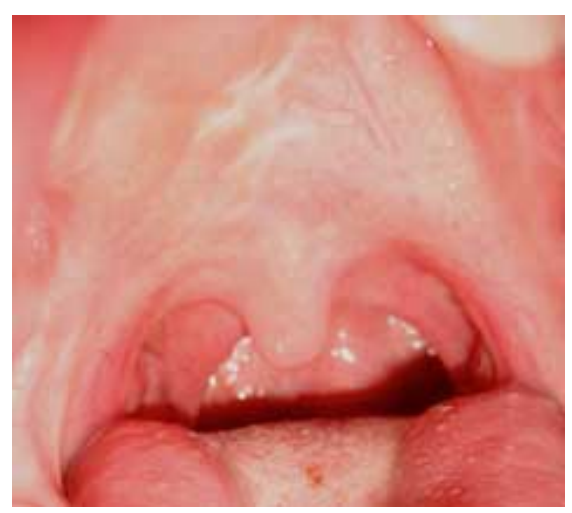

Fig. 1. Velo del paladar con morfología convexa.

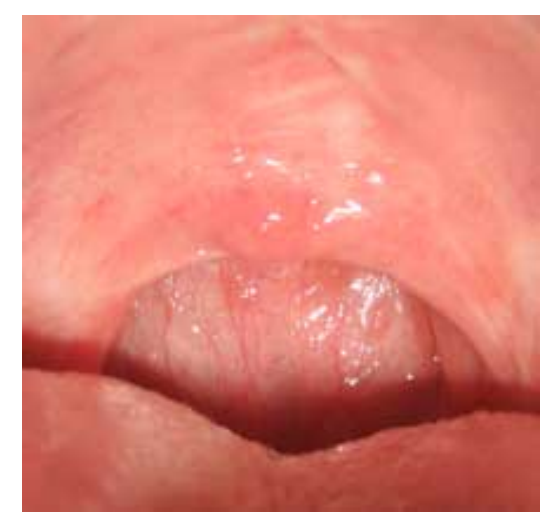

Fig. 2. Velo del paladar con morfología plana.

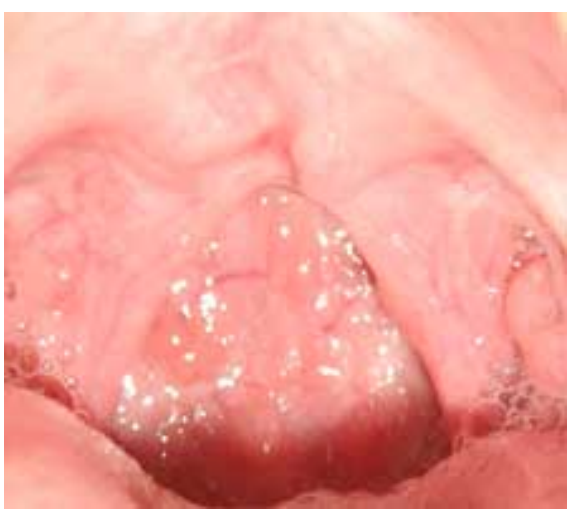

Fig. 3. Velo del paladar con morfología cóncava. 
casos) fisura de tipo labio-palatina bilateral y el $8 \%(2$ casos) fisura labio-velo-palatina completa operada.

Con respecto a las características anatómicas del velo palatino, se observó en el 68\% (17 casos) simetría siendo el resto asimétrico. En el 52\% (13 casos) posterior a la cirugía la úvula se encontraba presente.

La distribución de individuos según la clasificación morfológica del velo palatino quedó establecida del siguiente modo: El 52\% de los velos palatinos se clasificaron como cóncavos, el $24 \%$ de ellos se clasificó como convexo y el restante $24 \%$ como plano.

En relación a la evaluación de la insuficiencia velo faríngea realizada a los individuos en estudio se determinó que el $12 \%$ de ellos se presentaron como insuficientes, el $56 \%$ como límite insuficiente, el 24\% como límite suficiente y tan solo el $8 \%$ como suficiente.

Al aplicar el análisis estadístico de Kruscal Wallis con el fin de establecer si existen diferencias estadísticamente significativas entre la morfología del velo palatino y el puntaje obtenido en la evaluación velo faríngea, se estableció un $\mathrm{p}>0,05(\mathrm{p}=0,3)$.

\section{DISCUSIÓN}

Los músculos que estructuran el velo palatino corresponden al tensor del velo palatino, elevador del velo palatino, palatogloso, palatofarÍngeo y músculo de la úvula. Los cuatro primeros músculos se disponen en forma de arco siendo el músculo tensor y el elevador del velo palatino los de disposición superior, mientras que los músculos palatogloso y palatofaríngeo los de disposición inferior (Latarjet \& Ruiz-Liard; Rouvière \& Delmas).

El músculo tensor y elevador del paladar son músculos cuyo origen a nivel craneal se relacionan íntimamente con la porción cartilaginosa de la tuba auditiva, permitiendo su dilatación y por ende la descompresión del oído medio cada vez que se eleva y tensa el velo palatino (Rouvière \& Delmas). El músculo tensor del velo palatino desciende verticalmente desde su origen a nivel de la fosita navicular del proceso pterigoides del hueso esfenoides hasta reflejarse por medio de un tendón a nivel del gancho o hamulus pterigoideo, desde ese punto el tendón se horizontaliza y aplana, uniéndose con el tendón del lado contrario, de este modo el esqueleto del velo comienza a tomar forma. El resto de la musculatura se insertará en este esqueleto aponeurótico produciéndose la interdigitación de la musculatura del lado derecho e izquierdo. Esta situación presenta una excepción debido a que el músculo de la úvula se dispone horizontal con una dirección antero posterior a nivel de la línea media sobre la aponeurosis palatina (Latarjet \& Ruiz-Liard; Rouvière \& Delmas).

En cambio, en el paciente fisurado todas las relaciones anatómicas recién expuestas se pierden debido a la solución de continuidad del tejido duro y blando del techo de la cavidad oral, por lo cual los arcos anteriormente mencionados no se formarán y mas aún el tejido se colapsará, trasladara y retraerá, determinando la pérdida de la morfofunción velar (Cortés Araya et al.).

En el presente estudio se propuso una clasificación de la morfología que adquiere el velo palatino en el individuo fisurado posterior a la realización de los procedimientos quirúrgicos de cierre primario del paladar. Esto responde al hecho que no existe una clasificación de tipo clínico que pueda apoyar el diagnóstico y posteriormente el pronóstico funcional de la nueva estructura.

Si bien el numero de individuos evaluados corresponde a un número bajo de casos analizados, estos se distribuyen en relación al tipo de fisura de manera similar a lo establecido en otros estudio (Sepúlveda Troncoso et al.), siendo siempre el mas prevalente aquel de fisura labiopalatina unilateral $(76 \%)$.

El grado de simetría del velo (68\% de los casos en este estudio), si bien intenta demostrar una mayor armonización morfológica postquirúrgica, no es un factor que por sí refleje funcionalidad, mas bien, ésta ultima debiese ser definida en función de la morfología velar. De este modo, se establece que idealmente un velo postquirúrgico debiera ser convexo, simétrico y con permanencia del músculo úvula, con el objetivo de poder cerrar la comunicación entre oro y nasofaringe durante la elevación del mismo.

La morfología del velo palatino observada en este estudio dio cuenta que mas de la mitad de los casos (52\%) presentaron una morfología cóncava, lo que al ser sumado con el $24 \%$ de los individuos cuya morfología velar fue clasificada como plana, nos dice que $3 / 4$ de los velos analizados presentan una morfología alejada de los patrones normales de un velo-palatino y del gold standar anatómico a conseguir con el cierre quirúrgico del velo. Esto refleja en primer caso la dificultad de devolver a su posición las estructuras anatómicas mediante la cirugía y en función de esto, la no incorporación del músculo de la úvula en su disposición anatómica normal, siendo este músculo el que le otorgará el aspecto convexo del velo palatino. Si bien en este estudio en el $52 \%$ de los individuos fue posible obser- 
var la existencia del músculo de la úvula, una gran parte de ellos se encontraban atróficos o desplazados, no permitiendo otorgar la morfología convexa al velo.

La comunicación directa de la cavidad oral con la cavidad nasal, genera, una serie de alteraciones fonéticas, respiratorias, infecciosas e inflamatorias que afectan al sistema por completo (Lazzeri et al., 2008). Como la función muscular se ve alterada, la tracción que normalmente debe producir el velo palatino por sobre la tuba auditiva también se ve afectada, por lo cual comenzara a producirse el acúmulo de secreciones a nivel del oído medio, lo que posteriormente traerá como consecuencia una serie de sintomatología de tipo auditiva, empeorando aun mas el cuadro o condición de la malformación (da Silva et al.; Kwan et al.).

Las insuficiencias velo faríngeas se han definido como la incapacidad del velo palatino en la obturación del esfínter velo faríngeo (Filip et al., 2011; Ministerio de Salud; Ysunza et al., 2002) permitiendo el escape del aire hacia la cavidad nasal durante la emisión de los fonemas vocálicos y consonánticos, excepto para $\mathrm{m}, \mathrm{n}$ y ñ que son por naturaleza nasales (Cortés Araya et al.). Se ha propuesto como medida terapéutica para este trastorno la realización de una cirugía secundaria de velo palatino en conjunto con tratamiento fonoaudiológico.

Desde el punto de vista fonoaudiológico, los individuos fisurados y producto de la insuficiencia velo faríngea, se caracterizan por un retraso del lenguaje, alteraciones del habla y la voz, las que habitualmente se traducen en hipernasalidad, consonantes orales débiles y articulaciones compensatorias (Cortés Araya et al.; Ministerio de Salud; Nyberg et al.; Smith \& Guyette, 2004; Ysunza et al., 2011).

Los trastornos del lenguaje que afectan a los individuos fisurados, no son causados por la existencia de una fisura y por ende de una insuficiencia velo faríngea, si no que ésta predispone al aprendizaje de compensaciones articulatorias, de modo tal que se desarrollan patrones fonéticos anormales durante el desarrollo pre-lingüístico, que pueden adquirir patrones fonológicos inusuales (Ministerio de Salud); que al persistir en el tiempo, se incorporan en el sistema de reglas lingüísticas del niño/a conformando un retraso o trastorno del lenguaje.

Con el objetivo de evaluar el grado de disfunción a nivel del lenguaje observada en los individuos fisurados operados, y como variable subrogante a la misma se evaluó el grado de insuficiencia velo faríngea. La mayor parte de los individuos en estudio (56\%) presentó una evalua- ción de límite insuficiente, lo cual sumado a los individuos con una evaluación insuficiente alcanzan alrededor del 68\% del total de individuos estudiados. Estos datos demuestran el gran déficit funcional existente en los individuos fisurados operados, en donde si bien se logra el cierre entre cavidad nasal y oral, éste no logra ser de la calidad morfológica que le permita trabajar bajo parámetros funcionales normales. Esta situación suena aún mas preocupante si recordamos que en este estudio se excluyeron a aquellos pacientes con condiciones sindrómicas asociados a fisuras labio- palatinas. Los individuos cuyas fisuras se encuentran formando parte de entidades sindrómicas presentaran mayores alteraciones fonoaudiológicas, por lo que deberán ser considerados como de alto riesgo, con un abordaje fonoaudiológico precoz (Ministerio de Salud; Ysunza et al., 2011).

La morfología del velo palatino (cóncava, plana y convexa), no pudo relacionarse de forma estadísticamente significativa con el grado de evaluación velo faríngea ( $p>0,05)$, por lo tanto en este estudio se pudo establecer que la forma convexa del velo palatino no necesariamente determinará una mejor evaluación velo faríngea, lo cual es evidentemente contrario a lo que se pudiera suponer; del mismo modo un velo cóncavo no necesariamente tendrá una peor evaluación velo faríngea, vale decir, estas variables se comportan entre si de forma independiente. Una posible explicación a esta situación, es determinada por el modo en que se clasificó el velo palatino desde el punto de vista morfológico, ya que ésta se realizó con un velo pasivo y no durante la función de competencia velar. Esto nos hace pensar en un cambio morfológico del velo palatino en su transición de la pasividad a la activación, por lo cual el velo debiera clasificarse morfológicamente tanto en su etapa pasiva como en su etapa dinámica. Por otro lado, si bien la forma de las estructuras pueden dar respuesta a una determinada función, también lo será la variable tamaño de la misma, por lo cual si bien pueden existir velos convexos que homologuen lo observado en un velo de un individuo sano, estos pueden carecer del tamaño suficiente para obliterar el hiato velo-faríngeo manteniéndose la comunicación y por ende una deficiente evaluación velo-faríngea.

La correcta y oportuna evaluación de la insuficiencia velo faríngea, permitirá establecer y categorizar el grado de afección que ésta produce en la calidad de la fonoarticulación. Para ello se cuenta con el estudio clínico de la insuficiencia sumado a una serie de exámenes que complementaran el diagnóstico fonoaudiológico, para establecer las medidas terapéuticas adecuadas. Dentro de estos procedimientos se pueden incluir videofluoroscopía y la nasofaringoscopía (Lipira et al., 2011; Ministerio de Salud; Ysunza et al., 2011). 


\section{CONCLUSIONES}

El velo palatino puede clasificarse en individuos fisurados operados según su morfología en velos cóncavos, planos y convexos. Un gran porcentaje de los individuos en estudio presentaron bajos valores en su evaluación velo faríngea 68\% (entre insuficientes y límite insuficiente). Por otro lado no existió una relación estadísticamente significati- va entre la morfología del velo y el grado de insuficiencia velo faríngea $(p>0,05)$. Si bien en este estudio no se estableció la ya citada relación, es un primer acercamiento a futuras investigaciones en donde la evaluación morfológica sea realizada en una etapa activa y apoyándose en otras herramientas tales como la videofluoroscopía y la nasofaringoscopía.

HERNANDEZ, C. A.; MUÑOZ, D. M; SALAZAR, P. C. \& LEIVA, V. N. Palate velum morphology on operated cleft palate patients and its relation with the degree of pharyngeal velum insufficiency. Int. J. Morphol., 31(1):87-93, 2013.

SUMMARY: The reason for lip and cleft palate is due to a congenital malformation characterized by the interruption in the continuity of tissue. The primary surgery of the palate velum allows us to reconstruct the lost morphology in the velum palate, however this doesn't always consider the return of the uvula muscle anatomy, whereby the operated velum becomes an anomalous anatomy thus preventing an optimum closing between the nasal and oral cavity; this condition is known as pharyngeal velum insufficiency. The aim was to associate palate velum morphology with pharyngeal velum insufficiency on cleft palate operated patients. An analytical study was done, using the velum palate as sample; the sample was selected amongst patients who met the inclusion criteria. A photographic record and a clinical exam were done on those patients who were chosen in order to classify the anatomy of the velum palate. Subsequently a phonoaudiological evaluation was done in order to establish the degree of pharyngeal velum insufficiency. The data obtained were statistically tabulated and evaluated. The morphological classification of the palate velum determined that $52 \%$ of the vela were classified as concave; the flat and convex vela both had a $24 \%$ share each. According to the evaluation of the pharyngeal velum, $12 \%$ were shown to be insufficient, $52 \%$ as borderline insufficient, $24 \%$ as borderline sufficient, and only $8 \%$ proved to be sufficient. When relating the palate velum morphology with pharyngeal velum evaluation a $\mathrm{p}=0.3$ was obtained. There is a variability in both the palate velum morphology and in the evaluation of the pharyngeal velum insufficiency on operated cleft palate patients, with the latter shown as being unfavorable. No statistical relation was established between palate velum morphology and pharyngeal velum evaluation.

KEY WORDS: Lip and cleft palate; Velum palatine anatomy; Pharyngeal velum evaluation.

\section{REFERENCIAS BIBLIOGRÁFICAS}

Carinci, F.; Scapoli, L.; Palmieri, A.; Zollino, I. \& Pezzetti, F. Human genetic factors in nonsyndromic cleft lip and palate: an update. Int. J. Pediatr. Otorhinolaryngol., 71(10):1509-19, 2007.

Cortés Araya, J.; Niño Duarte, A. Y.; Sung Hsieh, H. H. \& Gómez Sánchez, B. Veloplastia funcional secundaria: Una alternativa no obstructiva en el tratamiento de la insuficiencia velofaríngea. Rev. Esp. Cirug. Oral y Maxilofac., 26(2):110-9, 2004.

da Silva, D. P.; Collares, M. V. \& da Costa, S. S. Effects of velopharyngeal dysfunction on middle ear of repaired cleft palate patients. Cleft Palate Craniofac. J., 47(3):225-33, 2010.

Filip, C.; Matzen, M.; Aagenæs, I.; Aukner, R.; Kjøll, L.; Høgevold, H. E.; Abyholm, F. \& Tønseth, K. Speech and magnetic resonance imaging results following autologous fat transplantation to the velopharynx in patients with velopharyngeal insufficiency. Cleft Palate Craniofac. J., 48(6):708-16, 2011.

Kwan, W. M.; Abdullah, V. J.; Liu, K.; van Hasselt, C. A. \& Tong, M. C. Otitis media with effusion and hearing loss in Chinese children with cleft lip and palate. Cleft Palate Craniofac. J., 48(6):684-9, 2011.

Latarjet, M. \& Ruiz-Liard, A. Anatomía Humana. $3^{\mathrm{a}}$ ed. Buenos Aires, Médica Panamericana, 1995.

Lazzeri, D.; Viacava, P.; Pollina, L. E.; Sansevero, S.; Lorenzetti, F.; Balmelli, B.; Funel, N.; Gatti, G. L.; Naccarato, A. G. \& Massei, A. Dystrophic-like alterations characterize orbicularis oris and palatopharyngeal muscles in patients affected by cleft lip and palate. Cleft Palate Craniofac. J., 45(6):587-91, 2008.

Lipira, A. B.; Grames, L. M.; Molter, D.; Govier, D.; Kane, A. A. \& Woo, A. S. Videofluoroscopic and nasendoscopic correlates of speech in velopharyngeal dysfunction. Cleft Palate Craniofac. J., 48(5):550-60, 2011.

Lorente, C.; Cordier, S.; Goujard, J.; Aymé, S.; Bianchi, F.; Calzolari, E.; De Walle, H. E. \& Knill-Jones, R. Tobacco and alcohol use during pregnancy and risk of oral clefts. Occupational Exposure and Congenital Malformation Working Group. Am. J. Public Health, 90(3):415-9, 2000. 
Ministerio de Salud. Guía Clínica Fisura Labiopalatina. Santiago, Minsal, 2009.

Mulliken, J. B.; Wu, J. K. \& Padwa, B. L. Repair of bilateral cleft lip: review, revisions, and reflections. J. Craniofac. Surg., 14(5):609-20, 2003.

Nanda, V.; Tuli, P. \& Sharma, R. K. Lateral approach to the levator veli palatini: a preliminary report. Cleft Palate Craniofac. J., 46(3):292-4, 2009.

Nyberg, J.; Westberg, L. R.; Neovius, E.; Larson, O. \& Henningsson, G. Speech results after one-stage palatoplasty with or without muscle reconstruction for isolated cleft palate. Cleft Palate Craniofac. J., 47(1):92-103, 2010.

Palomino, H. M.; Palomino, H.; Cauvi, D.; Barton, S. A. \& Chakraborty, R. Facial clefting and amerindian admixture in populations of Santiago, Chile. Am. J. Hum. Biol., 9(2):225$32,1997$.

Pardo, R.; Nazer, J. \& Cifuentes, L. Prevalencia al nacimiento de malformaciones congénitas y de menor peso de nacimiento en hijos de madres adolescentes. Rev. Med. Chile, 131(10):116572, 2003.

Rogers, C. R.; Weinberg, S. M.; Smith, T. D.; Deleyiannis, F. W.; Mooney, M. P. \& Marazita, M. L. Anatomical basis for apparent subepithelial cleft lip: a histological and ultrasonographic survey of the orbicularis oris muscle. Cleft Palate Craniofac. J., 45(5):518-24, 2008.

Rojas, A.; Ojeda, M. E. \& Barraza, X. Malformaciones congénitas y exposición a pesticidas. Rev. Med. Chil., 128(4):399-404, 2000 .

Rouvière, H. \& Delmas, A. Anatomía Humana descriptiva, topográfica y funcional. Tomos 1 y $4.11^{\mathrm{a}}$ ed. Barcelona, Masson, 2005.

Sepúlveda Troncoso, G.; Palomino Zúñiga, H. \& Cortés Araya, J. Prevalencia de fisura labiopalatina e indicadores de riesgo: Estudio de la población atendida en el Hospital Clínico Félix Bulnes de Santiago de Chile. Rev. Esp. Cir. Oral y Maxilofac., 30(1):17-25, 2008.

Shaw, G. M.; Nelson, V.; Iovannisci, D. M.; Finnell, R. H. \& Lammer, E. J. Maternal occupational chemical exposures and biotransformation genotypes as risk fac- tors for selected congenital anomalies. Am. J. Epidemiol., 157(6):475-84, 2003.

Smith, B. \& Guyette, T. W. Evaluation of cleft palate speech. Clin. Plast. Surg., 31(2):251-60, 2004.

Stuppia, L.; Capogreco, M.; Marzo, G.; La Rovere, D.; Antonucci, I.; Gatta, V.; Palka, G.; Mortellaro, C. \& Tetè, S. Genetics of syndromic and nonsyndromic cleft lip and palate. J. Craniofac. Surg., 22(5):1722-6, 2011.
Ysunza, A.; Carmen Pamplona, M. \& Santiago Morales, M. A. Velopharyngeal valving during speech, in patients with velocardiofacial syndrome and patients with non-syndromic palatal clefts after surgical and speech pathology management. Int. J. Pediatr. Otorhinolaryngol., 75(10):1255-9, 2011.

Ysunza, A.; Pamplona, C.; Ramirez, E.; Molina, F.; Mendoza, M. $\&$ Silva, A. Velopharyngeal surgery: a prospective randomized study of pharyngeal flaps and sphincter pharyngoplasties. Plast. Reconstr. Surg., 110(6):1401-7, 2002.

Dirección para Correspondencia

Prof. Dr. Arnoldo Alejandro Hernández Caldera

Instituto de Ciencias Biomédicas

Facultad de Medicina

Universidad de Chile

Santiago

CHILE

Email: arnoldohernandezc@med.uchile.cl

Recibido : 12-06-2012

Aceptado: 10-09-2012 